\title{
Propiedades mecánicas de resinas compuestas modificadas o no con poliácidos
}

\author{
Ruiz JM*, Ceballos L**, Fuentes $\mathrm{MV}^{* * *}$, Osorio R****, Toledano $\mathrm{M}^{* * * *}$, \\ García-Godoy F*****
}

RESUMEN

Objetivo: Determinar la resistencia a la flexión y el módulo de elasticidad de cinco resinas compuestas: Tetric Ceram, Ecusit, Spectrum TPH, Filtek Z-250, Degufill mineral; y dos resinas modificadas con poliácidos: Luxat e Ionosit. Material y método: Se prepararon cinco muestras de cada material en un molde metálico rectangular (22x2x2 mm). Las muestras fueron polimerizadas, primero en el centro y luego en ambos extremos con una lámpara de luz halógena y después se conservaron en agua a $37^{\circ} \mathrm{C}$ durante 48 horas. Las muestras se pulieron para eliminar la capa de resina superficial y se sometieron al ensayo de flexión en 3 puntos con una máquina de tracción universal Instron con una velocidad de travesaño de $1 \mathrm{~mm} / \mathrm{min}$. Los resultados $(\mathrm{MPa})$ fueron analizados mediante los tests de ANOVA y Student-Newman-Keuls $(p<0.05)$. Resultados: La resistencia a la flexión y el módulo de elasticidad de la resina compuesta modificada con poliácidos lonosit fueron significativamente menores que los determinados para los demás materiales evaluados.

Palabras clave: Propiedades mecánicas, resistencia a la flexión, módulo de elasticidad, resina compuesta, resina compuesta modificada con poliácido.

\begin{abstract}
The aim of this study was to determine the flexural strength and modulus of elasticity of five resin composites: Tetric Ceram, Ecusit, Spectrum TPH, Filtek Z-250, Degufill mineral; and two polyacid-modified resin composites:

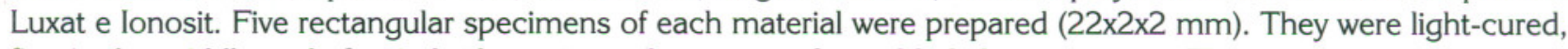
first in the middle and after in both extremes, by means of a visible-light curing unit. The specimens were stored in water at $37^{\circ} \mathrm{C}$ for 48 hours and ground to eliminate the resin layer inhibited by oxygen. Three-point bending test was performed in an universal testing machine (Instron) at a cross-head speed of $1 \mathrm{~mm} / \mathrm{min}$. Results were expressed in MPa and analysed by ANOVA and Student-Newman-Keuls tests $(\mathrm{p}<0.05)$. Flexural strength and modulus of elasticity of the polyacid-modified resin composite, Ionosit, were significantly lower than the values obtained for the rest of the materials evaluated.

Key words: Mechanical properties, flexural strength, modulus of elasticity, resin composite, polyacid-modified resin composite

* $\quad$ Estudiante de la Facultad de Odontología y Becaria de Investigación del Plan Propio de la Universidad de Granada.

** Becaria Post-doctoral del Plan Propio de la Universidad de Granada, adscrita al Dpto. de Estomatología.

*** Licenciada en Odontología.

**** Profesor Titular de Materiales Odontológicos. Facultad de Odontología. Universidad de Granada.

****** Profesor y Director de Investigación del College of Dental Medicine, Nova Southeastern University, Fort Lauderdale, FL, Estados Unidos.
\end{abstract}

Ruiz JM, Ceballos L, Fuentes MV, Osorio R, Toledano M, García-Godoy F. Propiedades mecánicas de resinas compuestas modificadas o no con poliácidos. Av. Odontoestomatol 2003; 19-6: 291-297. 


\section{INTRODUCCIÓN}

Las mejoras en las propiedades de las resinas compuestas han permitido que sean el material restaurador actualmente más utilizado en dientes permanentes. No obstante, la propiedad anticariogénica de los cementos de ionómero de vidrio debido a la liberación de flúor, y su capacidad para adherirse químicamente al tejido dentario $(1,2,3)$, hacen que estos materiales sean aún muy atractivos para el clínico (4). Sobre todo, si tenemos en cuenta que la caries secundaria es el motivo principal para reemplazar las restauraciones antiguas (5).

Sin embargo, los cementos de ionómero de vidrio presentan algunas desventajas en comparación con las resinas compuestas como son: un tiempo de trabajo corto y un tiempo de fraguado largo, sorción y solubilidad elevadas, peores propiedades estéticas (3) y, fundamentalmente, una baja resistencia a la fractura $(4,6,7,8)$ y al desgaste (7). Debido a estas propiedades mecánicas deficientes, los cementos de ionómero de vidrio no están indicados para ser utilizados en restauraciones sometidas a carga, como son las de Clase I y II $(4,9)$.

Con el fin de conseguir un material con unas propiedades mecánicas similares a las de las resinas compuestas, pero manteniendo las características beneficiosas de los cementos de ionómero de vidrio se desarrollaron materiales híbridos: los cementos de ionómero de vidrio modificados con resina y las resinas modificadas con poliácidos, conocidas como compómeros $(8,10,11)$. Estos materiales estarían indicados para pacientes con un alto riesgo de caries y fueron diseñados para restaurar lesiones de Clase I, III y V (12).

Las magnitudes fundamentales para conocer la resistencia a las fuerzas oclusales de un material determinado son: la resistencia a la flexión, el módulo de elasticidad y la resistencia a la fractura (13). Por tanto, su cuantificación permite también predecir la aparición de fracturas tanto en el propio material como en los márgenes (14) de la restauración, o lo que es lo mismo, la longevidad de la restauración bajo condiciones clínicas simuladas (11).

Algunos trabajos han demostrado que las propieda- des físicas de estos materiales híbridos son muy superiores a las de los cementos de ionómero de vidrio, siendo, en el caso de algunas resinas modificadas con poliácidos comercializadas, ligeramente inferiores a las de las resinas compuestas $(9,12) y$, en el caso de otras, prácticamente similares (12).

El objetivo del presente estudio fue evaluar el comportamiento mecánico, por medio de la determinación de la resistencia y del módulo de elasticidad, de cinco resinas compuestas y de dos resinas modificadas con poliácidos disponibles en el mercado.

\section{MATERIAL Y MÉTODO}

En el presente estudio se evaluaron cinco resinas compuestas: Tetric Ceram (Vivadent, Liechtenstein), Ecusit (DMG, Hamburgo, Alemania), Degufill mineral (Degussa Dental GmbH, Hanau, Alemania), Spectrum TPH (Dentsply-Detrey GmbH, Konstanz, Alemania) y Filtek Z-250 (3M-ESPE, St. Paul, MN, EE.UU.); y dos resinas modificadas con poliácidos: Luxat (DMG, Hamburgo, Alemania) e IonositBaseliner (DMG, Hamburgo, Alemania).

Las propiedades de flexión de los materiales se determinaron mediante un test de flexión en tres puntos de acuerdo con las normas ISO 4049 (15). Se prepararon cinco barritas de cada material utilizando un molde rectangular metálico con las siguientes dimensiones: $22 \mathrm{~mm}$ de longitud, $2 \mathrm{~mm}$ de anchura y $2 \mathrm{~mm}$ de espesor. Los moldes se colocaron sobre una loseta de vidrio y el material de estudio se insertó procurando que no quedaran burbujas de aire atrapadas en su interior. Sobre éste se colocó una banda matriz de acetato y se hizo presión con otra loseta de vidrio para conseguir una superficie lisa y un grosor homogéneo. Los especímenes fueron polimerizados con una lámpara de luz halógena (Spectrum 800, Dentsply. Detrey Gmb H, Konstanz, Alemania), primero en el centro y después en los extremos, teniendo cada exposición una duración de 40 segundos. Cada diez exposiciones se comprobó que la densidad de potencia de la lámpara sobrepasase los 600 $\mathrm{mw} / \mathrm{cm}^{2}$ con un radiómetro de curado que lleva incorporado la lámpara. 
Los especímenes se conservaron en agua destilada a $37^{\circ} \mathrm{C}$ durante 48 horas y transcurrido ese tiempo se pulieron con discos de carburo de silicio de 500 grit para eliminar la capa de resina inhibida por oxígeno. Las dimensiones de los especímenes fueron medidas de nuevo mediante un calibre digital (Mitutoyo, Tokio, Japón).

Los especímenes fueron sometidos al ensayo de flexión en tres puntos en una máquina de tracción universal Instron (Modelo 4411, Instron Corp., Canton, MA, EE.UU.) con una velocidad de travesaño de 1 $\mathrm{mm} / \mathrm{min}$. Durante el ensayo las barritas están apoyadas en dos puntos. La carga se aplica en la superficie opuesta a los puntos de apoyo en un punto equidistante de los dos anteriores. En la zona cóncava se producen fenómenos de compresión, mientras que en la zona convexa se aprecian fenómenos de tensión, hasta que se produce la fractura de la barrita.

Los resultados obtenidos se analizaron mediante el test de ANOVA para determinar si las propiedades de flexión dependían del material de estudio. El nivel de significación establecido previamente fue de $\mathrm{p}<0.05$. Las comparaciones múltiples posteriores se realizaron con el test de Student-Newman-Keuls. Todos los datos se analizaron con el programa SPSS 10.0 para Windows software (SPSS Inc., Chicago, IL, EE.UU.).

\section{RESULTADOS}

Las medias (x) y desviaciones típicas (dt) de los distintos materiales quedan reflejadas en la tabla 1.

El test de ANOVA puso de manifiesto la influencia del material evaluado en las variables dependientes resistencia a la flexión y módulo de elasticidad $(\mathrm{F}=21.41, \mathrm{p}<0.0001 ; \mathrm{F}=8.21, \mathrm{p}<0.001$, respectivamente). En el caso de la resistencia a la flexión, las comparaciones múltiples con el test de StudentNewman-Keuls permitieron ordenar los materiales del siguiente modo: Spectrum TPH presentó unos valores similares a los de Filtek Z-250 y Luxat, y superiores a los de Ecusit, Degufill mineral, Tetric Ceram e Ionosit-Baseliner. Filtek Z-250, Luxat, Ecusit y Degufill mineral mostraron una resistencia a la flexión similar. Los valores obtenidos por Tetric Ceram fueron similares a los de Luxat, Ecusit y Degufill, pero significativamente inferiores a los de Spectrum TPH y Filtek Z-250. La resina modificada con poliácido lonosit-Baseliner presentó una resistencia a la flexión significativamente más baja que la del resto de los materiales.

En cuanto al módulo de elasticidad, la resina compuesta modificada con poliácidos, Luxat, fue el material que presentó los valores más altos, siendo similares a los de todas las resinas compuestas evaluadas, con excepción del Tetric Ceram. IonositBaseliner presentó un módulo de elasticidad significativamente más bajo que el del resto de los materiales evaluados.

\section{DISCUSIÓN}

Se han descrito muchos métodos estáticos y dinámicos para determinar las propiedades mecánicas de los materiales resinosos (13). Dentro de los estáticos, el test de flexión en tres puntos descrito por la ISO-4049 (15), es el más utilizado (13) y permite eva-

\begin{tabular}{|c|c|c|c|c|c|c|c|c|}
\hline \multicolumn{1}{|c|}{ TABLA 1.- RESISTENCIA A LA FLEXIÓN Y MÓDULO DE ELASTICIDAD DE LOS DIFERENTES } \\
\hline MATERIALES EVALUAADOS. MEDIAS (dt) EN MPa \\
\hline MATERIAL & SPECTRUM & Z-250 & LUXAT & ECUSIT & DEGUFILL & T. CERAM & LONOSIT \\
\hline Resistencia a & $140.1 \mathrm{a}$ & $130.1 \mathrm{ab}$ & $116.9 \mathrm{abc}$ & $108.2 \mathrm{bc}$ & $104.9 \mathrm{bc}$ & $97.6 \mathrm{c}$ & $33.1 \mathrm{~d}$ \\
la flexión & $(18.7)$ & $(6.9)$ & $(16.6)$ & $(14.1)$ & $(23.3)$ & $(9.5)$ & $(17.1)$ \\
\hline Módulo de & $10972.0 \mathrm{a}$ & $10874.3 \mathrm{ab}$ & $12060.8 \mathrm{a}$ & $9702.6 \mathrm{ab}$ & $10069.8 \mathrm{ab}$ & $8354.5 \mathrm{~b}$ & $4930.7 \mathrm{c}$ \\
elasticidad & $(746.2)$ & $(1927.9)$ & $(2626.6)$ & $(2306.0)$ & $(1309.2)$ & $(1248.8)$ & $(975.5)$ \\
\hline
\end{tabular}

Medias con las mismas letras indican que no hay diferencias estadísticamente significativas $(p>0.05)$. 
luar simultáneamente la resistencia a la flexión y el módulo de elasticidad.

La resistencia a la flexión se define como la carga máxima a la que el material se fractura (11). En el presente estudio, la resina compuesta híbrida Spectrum TPH presentó la resistencia a la flexión más elevada. Este material ha demostrado un buen comportamiento mecánico en otros estudios (16, 17 ), incluso cuando se ha comparado con resinas compactables, cuyas propiedades mecánicas se suponen mejores (17) por su mayor contenido en relleno. Además, sus partículas son irregulares y eso hace que la resistencia a la flexión sea mejor que las de los materiales que incorporan partículas redondas o pre-polimerizadas (16).

Las otras resinas compuestas evaluadas obtuvieron valores ligeramente inferiores, en un rango que oscila entre los 97.6 MPa y los $130.1 \mathrm{MPa}$, resultados que concuerdan con lo publicado previamente $(9,14,16,18)$.

Los factores que más influyen en las propiedades mecánicas de un material son su contenido en relleno, el tamaño de las partículas, su distribución y las interacciones entre el relleno y la matriz $(9,14,16$, 19), siendo el contenido en volumen del relleno la propiedad que más se correlaciona con la resistencia del material, con su módulo de elasticidad, así como con su resistencia a la fractura $(11,14)$. También se ha descrito que los composites que incorporan flúor soluble, presentan una resistencia a la flexión menor, porque el flúor se disuelve y quedan microporosidades que facilitan la propagación de cracks (9). Este sería el caso del Tetric Ceram, que incorpora vidrio de fluorosilicato de aluminio y bario, y, sobre todo, del Degufill mineral que tiene la propiedad, según sus fabricantes, de liberar de forma inteligente fluoruro, así como iones calcio y fosfato, que aportarían una protección del esmalte dental y reforzarían la remineralización natural.

De acuerdo con la literatura, las propiedades mecánicas de los materiales híbridos aumentan en relación a su contenido resinoso $(12,20,21)$. De este modo, las más bajas corresponderían a los cementos de ionómero de vidrio modificados con resina, serían mejores en el caso de las resinas modificadas con poliácidos y máximas para las resinas compuestas $(9,21)$.
Sin embargo, en el presente trabajo las dos resinas modificadas con poliácidos mostraron un comportamiento mecánico muy diferente. Ionosit-Baseliner mostró una resistencia a la flexión muy inferior, acercándose más los valores a los determinados en cementos de ionómero modificados con resinas (12). Sin embargo, la resistencia de Luxat fue similar a la de las resinas compuestas evaluadas, tal y como han determinado otros autores en el caso de otras resinas modificadas con poliácidos comercializadas (12).

En estos materiales se combinan componentes del cristal de polialquenoato típicos del cemento de ionómero de vidrio, por ejemplo, cristales de fluorosilicato, con sustancias polimerizables de las resinas compuestas, como son los dimetacrilatos (22). De este modo, contienen los componentes esenciales de los cementos de ionómero de vidrio, pero falta su reacción de fraguado ácido-base típica por la ausencia de agua en su composición $(3,23)$. Las diferencias obtenidas entre materiales del mismo tipo pueden deberse al grado en que han sido modificados con componentes más parecidos a las resinas o a los poliácidos. En el Ionosit-Baseliner sólo una pequeña porción de los grupos ácidos carboxílicos del ácido poliacrílico ha sido reemplazada por grupos metilmetacrilato lo que origina que se formen menos enlaces covalentes tras la polimerización, dando como resultado un material con propiedades más parecidas a las de los cementos de ionómero de vidrio, comparado con el Luxat (21).

La resistencia a la flexión de los materiales se correlaciona con su módulo de elasticidad, de tal forma que si la primera es alta el módulo de elasticidad lo será también (12). Esta relación se cumple en el presente trabajo, puesto que Ionosit-Baseliner, el material con la resistencia a la flexión más baja es también el material con el menor módulo de elasticidad. Similar situación acontece en el caso del Tetric Ceram, que fue la resina compuesta con los valores más bajos de ambas magnitudes. Los valores de módulo de elasticidad determinados para el resto de los materiales fueron prácticamente similares entre si, lo que concuerda con lo descrito por Peutzfeldt (20), quien encontró que las diferencias entre los módulos de elasticidad de diferentes materiales eran más pequeñas que entre los valores de resistencia a la flexión. 
El módulo de elasticidad describe la relación entre el estrés y la deformación que un material sufre para una carga determinada (24), es por tanto una medida de la rigidez relativa de un material (13). Un material más rígido requerirá una carga mayor para deformarse en el mismo grado que uno más elástico (24).

Idealmente, el módulo de elasticidad de los materiales ha de ser semejante al del tejido dentario. En las restauraciones de Clase $\mathrm{V}$ los materiales tienen que tener un módulo de elasticidad que les permita deformarse durante la flexión del diente, mientras que en las cavidades de Clase II el módulo de elasticidad debe ser lo suficientemente alto como para soportar las deformaciones y evitar la fractura de las cúspides (25). Sin embargo, el diente está compuesto fundamentalmente por esmalte y dentina, y ambos tejidos tienen un comportamiento elástico completamente diferente (24), por ello se asume que los materiales que se utilicen para restaurar dientes posteriores deben tener un módulo de elasticidad al menos igual al de la dentina o mayor, que está determinado en $18 \mathrm{GPa}(26,27)$. Además, cuanto menor sea la diferencia entre el módulo de elasticidad del material y la dentina, menor será la destrucción en la interfase material-dentina. Por tanto, esta magnitud juega un papel importante en la prevención de la microfiltración, la caries secundaria y la pérdida de las restauraciones $(28,29)$. Sin embargo, ninguno de los materiales evaluados presentó un módulo de elasticidad tan elevado como el determinado para la dentina, lo que coincide con los resultados descritos por otros autores $(13,16,18,24)$.

Las propiedades elásticas de un material van a condicionar su indicación clínica, lo que justifica que Ionosit-Baseliner sea utilizado únicamente como base cavitaria, con la ventaja de que su módulo de elasticidad bajo compense mejor el estrés de polimerización. En el caso de Luxat, sus propiedades mecánicas son similares a las de las resinas compuestas evaluadas, lo que permitiría su uso como material restaurador en zonas sometidas a carga. Sin embargo, es un material diseñado específicamente para restauraciones de Clase III y V. La razón es que existen otras propiedades de los materiales que hay que considerar como, por ejemplo, la sorción y solubilidad, que en el caso del Luxat es elevada comparada con la de las resinas compuestas, lo que supon- dría una mayor degradación del material con el tiempo y, por consiguiente, una reducción significativa de sus propiedades mecánicas.

De acuerdo con los resultados de este trabajo, las propiedades elásticas de los materiales estudiados coinciden con las de otros materiales comercializados y previamente evaluados, sin que en ningún caso, sean similares a las del tejido dentario que van a reemplazar. Los dos materiales clasificados como resinas compuestas modificadas con poliácidos presentaron un comportamiento mecánico completamente diferente entre sí, siendo el del Luxat comparable al de las resinas compuestas evaluadas.

\section{AGRADECIMIENTOS}

Al plan propio de la Universidad de Granada que concedió las Becas de Iniciación a la Investigación y la Beca Postdoctoral.

Los autores agradecen a Gertrudis GómezVillaescusa su ayuda en la preparación de los especímenes. Este trabajo fue financiado por el Proyecto de Investigación CICYT/FEDER MAT 2001-2843CO2, RED CYTED VIII. J.

\section{BIBLIOGRAFÍA}

1. Swift EJ, Pawlus MA, Vargas MA. Shear bond strengths of resin-modified glass-ionomer restorative materials. Oper Dent 1995; 20: 138-43.

2. Woolford MJ, Grieve AR. Release of fluorid from glass polyalkenoate (ionomer) cement subjected to radiant heat. J Dent 1995; 23: 233-7.

3. Kugel G. Direct and indirect adhesive restorative materials: A review. Am J Dent 2000; 13: 35D40D.

4. Xie D, Brantley WA, Culbertson BM, Wang G. Mechanical properties and microstructures of glass-ionomer cements. Dent Mater 2000; 16: 129-38. 
5. Mjör IA,Moorhead JE, Dahl JE. Reasons for replacement of restorations in permanent teeth in general dental practice. Int Dent J 2000; 50: 360-6.

6. McKinney JE, Antonucci JM, Rupp NW. Wear and microhardness of glass-ionomer cements. J Dent Res 1987; 66: 1134-9.

7. Croll TP. Glass ionomers for infants, children and adolescents. J Am Dent Assoc 1990; 120: 65-8.

8. Sidhu S.K, Watson T.F. Resin-modified glass ionomer materials. Am J Dent 1995; 8: No.1. 59-67.

9. Iazzetti G, Burgess JO, Gardiner D. Selected mechanical properties of fluoride-releasing restorative materials. Oper Dent 2001; 26: 21-6.

10. Iwami Y, Yamamoto H, Sato W, Kawai K, Torii M, Ebisu S. Weight change of various light-cured restorative materials after water immersion. Oper Dent 1998; 23: 132-7.

11. Gladys S, Van Meerbeek B, Braem M, Lambrechts P, Vanherle G. Comparative physicomechanical characterization of new hybrid restorative materials with conventional glass-ionomer and resin composite restorative materials. J Dent Res 1997; 76: 883-94.

12. Attin T, Vataschki M, Hellwig E. Properties of resin-modified glass-ionomer restorative materials and two polyacid-modified resin composite materials. Quintessence Int 1996; 27: 203-9.

13. Sabbagh J, Vreven J, Leloup G. Dynamic and static moduli of elasticity of resin-based materials. Dent Mater 2002; 64-71.

14. Manhart J, Kunzelmann KH, Chen HY, Kickel R. Mechanical properties and wear behavior of lightcured packable composite resins. Dent Mater 2000; 16: 33-40.

15. ISO-4049, Resin-based filling materials (1988).

16. Kim KH, Ong JL, Ojuno O. The effect of filler loading and morphology on the mechanical properties of contemporary composites. J Prosthet Dent 2002; 87: 642-9.

17. Cobb DS, MacGregor KM, Vargas MA, Denehy GE. The physical properties of packable and conventional posterior resin-based composites: a comparison. J Am Dent Assoc 2000; 131: 1610-5.

18. Hasegawa T, Itoh $\mathrm{K}$, Koike $\mathrm{T}$, Yukitani $\mathrm{W}$, Hisamitsu H, Wakumoto S, Fujishima A. Effect of mechanical properties of resin composites on the efficacy of the dentin bonding system. Oper Dent 1999; 24: 323-30.

19. Kalliyana Krishnan V, Yamuna V. Effect of initiator concentration, exposure time and particle size of the filler upon the mechanical properties of a light-curing radiopaque dental composite. J Oral Rehabil 1998; 25: 747-51.

20. Peutzfeldt A. Compomers and glass ionomers: Bond strength to dentin and mechanical properties. Am J Dent 1996; 9: 259-63.

21. El-Kalla IH, García-Godoy F. Mechanical properties of compomer restorative materials. Oper Dent 1999; 24: 2-8.

22. Geurtsen W, Leyhausen G, García-Godoy F. Effect of storage media on the fluoride relase and surface microhardness of four polyacid-modified composite resins ("compomers"). Dent Mater 1999; 15: 196-201.

23. McLean JW, Nicholson JW, Wilson AD. Proposed nomenclature for glass-ionomer dental cements and related materials. Quintessence Int 1994; 25: 587-9.

24. Abe Y, Lambrechts $P$, Inoue $S$, Braem MJA, Takeuchi M, Vanherle G, Van Meerbeek B. Dynamic elastic modulus of "packable" composites. Dent Mater 2001; 17: 520-5.

25. Xu HHK, Smith DT, Jahanmir S, Romberg E, Kelly JR, Thompson VP, Rekow ED. Indentation damage and mechanical properties of human enamel and dentin. J Dent Res 1998; 77: 472-80. 
26. Craig GR, Peyton FA. Elastic and mechanical properties of human dentin. J Dent Res 1958; 37: 710-8.

27. Willems G, Lambrechts P, Braem M, Celis JP, Vanherle G. A classification of dental composites according to their morphological and mechanical characteristics. Dent Mater 1992; 8: 310-9.

28. Montes MAJR, de Goes MF, Bernardi da Cunha MR, Soares $\mathrm{AB}$. A morphological and tensile bond strength evaluation of an unfilled adhesive with low-viscosity composites and a filled adhesive in one and two coats. J Dent 2001; 29: 435-41.

29. Labella R, Lambrechts P, Van Meerbeek B, Vanherle G. Polymerization shrinkage and elasticity of flowable composites and filled adhesives. Dent Mater 1999; 15: 128-37.

\section{CORRESPONDENCIA}

Dra. Laura Ceballos

C/ Emperatriz Eugenia 19, 6º

18003 Granada

e-mail: lauraceballos@hotmail.com

Tlf: 958372524 